\title{
RESEARCH
}

Open Access

\section{Genetic and behavioral adaptation of Candida parapsilosis to the microbiome of hospitalized infants revealed by in situ genomics, transcriptomics, and proteomics}

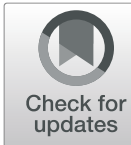

Patrick T. West ${ }^{1}$, Samantha L. Peters ${ }^{2,3}$, Matthew R. Olm ${ }^{4}$, Feiqiao B. Yu ${ }^{5}$, Haley Gause ${ }^{6}$, Yue Clare Lou' Brian A. Firek', Robyn Baker ${ }^{8}$, Alexander D. Johnson ${ }^{4,6}$, Michael J. Morowitz', Robert L. Hettich ${ }^{2,3}$ and Jillian F. Banfield ${ }^{5,9,10,11^{*} \text { (D) }}$

\begin{abstract}
Background: Candida parapsilosis is a common cause of invasive candidiasis, especially in newborn infants, and infections have been increasing over the past two decades. C. parapsilosis has been primarily studied in pure culture, leaving gaps in understanding of its function in a microbiome context.

Results: Here, we compare five unique C. parapsilosis genomes assembled from premature infant fecal samples, three of which are newly reconstructed, and analyze their genome structure, population diversity, and in situ activity relative to reference strains in pure culture. All five genomes contain hotspots of single nucleotide variants, some of which are shared by strains from multiple hospitals. A subset of environmental and hospital-derived genomes share variants within these hotspots suggesting derivation of that region from a common ancestor. Four of the newly reconstructed C. parapsilosis genomes have 4 to 16 copies of the gene RTA3, which encodes a lipid translocase and is implicated in antifungal resistance, potentially indicating adaptation to hospital antifungal use. Time course metatranscriptomics and metaproteomics on fecal samples from a premature infant with a $C$. parapsilosis blood infection revealed highly variable in situ expression patterns that are distinct from those of similar strains in pure cultures. For example, biofilm formation genes were relatively less expressed in situ, whereas genes linked to oxygen utilization were more highly expressed, indicative of growth in a relatively aerobic environment. In gut microbiome samples, C. parapsilosis co-existed with Enterococcus faecalis that shifted in relative abundance over time, accompanied by changes in bacterial and fungal gene expression and proteome composition.
\end{abstract}

Conclusions: The results reveal potentially medically relevant differences in Candida function in gut vs. laboratory environments, and constrain evolutionary processes that could contribute to hospital strain persistence and transfer into premature infant microbiomes.

Keywords: Microbial eukaryotes, Metagenomics, Genome-resolved metagenomics, Strain-tracking, Hospital microbiome, Neonatal intensive care unit, Premature infants, Candida

\footnotetext{
* Correspondence: jbanfield@berkeley.edu

${ }^{5}$ Chan Zuckerberg Biohub, San Francisco, CA, USA

${ }^{9}$ Department of Earth and Planetary Science, University of California, Berkeley,

CA, USA

Full list of author information is available at the end of the article
}

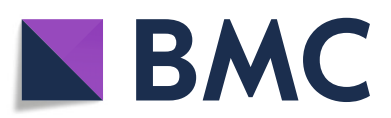

(- The Author(s). 2021 Open Access This article is licensed under a Creative Commons Attribution 4.0 International License, which permits use, sharing, adaptation, distribution and reproduction in any medium or format, as long as you give appropriate credit to the original author(s) and the source, provide a link to the Creative Commons licence, and indicate if changes were made. The images or other third party material in this article are included in the article's Creative Commons licence, unless indicated otherwise in a credit line to the material. If material is not included in the article's Creative Commons licence and your intended use is not permitted by statutory regulation or exceeds the permitted use, you will need to obtain permission directly from the copyright holder. To view a copy of this licence, visit http://creativecommons.org/licenses/by/4.0/ The Creative Commons Public Domain Dedication waiver (http://creativecommons.org/publicdomain/zero/1.0/) applies to the data made available in this article, unless otherwise stated in a credit line to the data. 


\section{Background}

Candida species are the most common cause of invasive fungal disease $[1,2]$. A variety of Candida species cause candidiasis and are recognized as a serious public health challenge, especially among immunocompromised and hospitalized patients [3, 4]. Historically, Candida albicans most commonly has been recognized as the cause of candidiasis, and as a result, is the focus of the majority of Candida research [4-6]. However, Candida parapsilosis, despite being considered less virulent than $C$. albicans, is the Candida species with the largest increase in incidence since 1990 [6]. Given important differences in the biology of $C$. albicans compared to non-albicans species, more research on non-albicans Candida species, especially the subset that poses a serious health risk, is needed [4].

C. parapsilosis is often a commensal member of the gastrointestinal tract and skin [6, 7]. Passage from hospital workers' hands to immunocompromised patients is thought to be a common cause of opportunistic infection in hospital settings [8]. C. parapsilosis infections of premature infants are of particular concern. Indeed, $C$. parapsilosis is the most frequently isolated fungal organism in many neonatal intensive care units (NICUs) in the UK [3] and is responsible for up to one-third of neonatal Candida bloodstream infections in North America [9]. Adding to the concern is the limited number of antifungal drugs and the increasing prevalence of antifungal drug resistance in Candida species. An estimated 3-5\% of $C$. parapsilosis are resistant to fluconazole, the most commonly applied antifungal [10]. The recent emergence of multidrug-resistant Candida auris with its resultant high mortality rate [11] serves as a warning regarding the potential for outbreaks of multidrugresistant $C$. parapsilosis. Therefore, understanding behavior of $C$. parapsilosis, both as a commensal organism and opportunistic pathogen, is incredibly important.

A challenge that complicates understanding of the medically relevant behavior of Candida in the human microbiome is that the hosts used in model infection systems (e.g., rat or murine mucosa) are not natural hosts to Candida species. Study of Candida in these models relies on some form of predisposition of the animal by occlusion, immunosuppression, surgical alteration, or elimination of competing microbial flora [1]. Pure culture experiments, an alternative to model system studies, are often the most accessible way to study Candida. However, the lack of a microbial community context is a large caveat, considering bacteria could influence the nutrition, metabolism, development, and evolution of eukaryotes. Indeed, other microbial eukaryotes have been shown to be dramatically influenced by their surrounding microbial communities. Choanoflagellates, the closest known living relative of animals, live in aquatic environments and feed on bacteria by trapping them in their apical collar [12]. The Choanoflagellate Salpingoeca rosetta is primarily a unicellular organism but formation of multicellular rosettes is induced by a sulphonolipd (RIF1) and inhibited by a sulfonatecontaining lipid, both produced by the bacterium Algoriphagus machipongonensis [13]. Furthermore, the bacterium Vibrio fischeri produces a chondroitinase, EroS, capable of inducing sexual reproduction in S. rosetta [14]. Together, these results demonstrate the influence that bacteria can exert on the morphology, development, and evolution of microbial eukaryotes.

There is more direct evidence motivating study of $C$. parapsilosis functioning in situ. For instance, Caenorhabditis elegans model of polymicrobial infection experiments showed that $C$. albicans exhibits complex interactions with Enterococcus faecalis, a bacterial human gut commensal and opportunistic pathogen. In this context, $C$. albicans and $E$. faecalis negatively impact one another's virulence [15], suggesting a mechanism that promotes commensal behavior in a gut microbial community context. The decrease in $C$. albicans virulence was attributed to inhibition of hyphal morphogenesis and biofilm formation by proteases secreted by $E$. faecalis [15] as well as E. faecalis capsular polysaccharide [16]. No research has investigated $C$. parapsilosis in a microbial community context.

An alternative to studying Candida species in animal models or laboratory cultures is to use an untargeted shotgun sequencing approach (genome-resolved metagenomics). DNA is extracted from fecal or other samples and sequenced. The subsequent DNA sequences are assembled, and metagenome-assembled genomes (MAGs) are reconstructed. Much work of this type has focused on the bacterial members of the human microbiome; however, recently developed methods such as EukRep [17] enable reconstruction of eukaryotic genomes from metagenomes with greater consistency, including genomes of Candida species [18]. The availability of genomes enables evolutionary studies and the application of other 'omics' approaches, such as transcriptomics, proteomics, and metabolomics, making it possible to go beyond metabolic potential to study activity in situ. Although there are limitations related to establishing causality via experimentation, the approaches can provide insights into metabolism and changes in metabolism linked to shifts in community composition in humanrelevant settings.

Here, we applied shotgun metagenomics, metatranscriptomics, and metaproteomics to investigate the behavior and evolution of Candida in the premature infant gut and hospital environment. Novel assembled C. parapsilosis and C. albicans genomes were reconstructed and the metagenomic data analyzed in terms of 
heterozygosity and population diversity. Due to the substantially less prior research on $C$. parapsilosis and the availability of $C$. parapsilosis-containing samples suitable for transcriptomics and proteomics, we focused our analyses on C. parapsilosis and identified genes and genomic regions under diversifying selection. Notably, we also identified instances of copy number gain of a gene involved in fluconazole resistance, pointing to a mechanism for hospital adaptation [19]. C. parapsilosis in situ transcriptomic and proteomic profiles were clearly distinct from profiles reported previously from culture settings. Substantial shifts in C. parapsilosis expression occurred with changes in microbiome composition over a few day period, suggesting the strong influence of bacterial community composition on $C$. parapsilosis behavior.

\section{Results}

\section{Recovery of novel Candida strain genomes}

A large dataset of a mixture of previously analyzed and newly generated infant gut and NICU shotgun metagenome samples were analyzed for the purpose of reconstructing novel Candida genomes (see the "Methods" section). Newly generated data includes fecal samples collected and sequenced from two infants targeted for having documented Candida infections during fecal collection. Previously analyzed data includes fecal samples collected from 163 premature infants primarily during the first 30 days of life (DOL) (full range of DOL 5121), with an average of 7 samples per infant. In addition, samples of the Neonatal intensive care unit (NICU) were taken from six patient rooms within the hospital housing the infants (Magee-Womens Hospital of UPMC, Pittsburgh, PA, USA). Finally, publicly available New York City subway shotgun metagenomes [20] were included after identifying Candida reads in one of the samples.

Candida genomes were assembled from samples containing $>2 \mathrm{Mbp}$ of predicted eukaryotic DNA using a EukRep-based pipeline [17]; see the "Methods" section for details. Eight new, unique Candida genomes were assembled for this study (Table 1), five C. albicans genomes, and three $C$. parapsilosis genomes. Three additional Candida genomes were assembled but have been analyzed previously [18] along with the bacterial component of the samples [21] (see the "Methods" section), totaling in 11 Candida genomes reconstructed from infant gut and hospital room metagenomes. Nine of the 11 genomes were reconstructed from premature infant fecal samples; 1 genome was derived from a NICU room sample S2_005, and 1 from New York City Subway Samples [20]. Genomes representing new strains

Table 1 Overview of Candida strain genomes used in this study

\begin{tabular}{|c|c|c|c|c|c|c|c|c|c|}
\hline Genome & Genus & Species & Length & $\begin{array}{l}\# \\
\text { Scaffolds }\end{array}$ & N50 & $\begin{array}{l}\text { BUSCO } \\
\text { comp. }\end{array}$ & $\begin{array}{l}\text { Year } \\
\text { sampled }\end{array}$ & Sample type & Reference \\
\hline C1_006 & Candida & Parapsilosis & 11852211 & 191 & 108686 & 92 & 2017 & Infant fecal metagenome & This study \\
\hline N3_182 & Candida & Parapsilosis & 12563647 & 342 & 65710 & 94 & 2013 & Infant fecal metagenome & $\begin{array}{l}\text { Olm et al. } 2019 \\
{[18,21]}\end{array}$ \\
\hline S2_005 & Candida & Parapsilosis & 11573959 & 1051 & 14507 & 93 & 2014 & NICU metagenome & $\begin{array}{l}\text { Olm et al. } 2019 \\
{[18,21]}\end{array}$ \\
\hline $\begin{array}{l}\text { NYC } \\
\text { Subway }\end{array}$ & Candida & Parapsilosis & 7420453 & 1285 & 6417 & 62 & NA & NYC subway metagenome & This study \\
\hline L2_023 & Candida & Parapsilosis & 4870205 & 2906 & 1700 & 35 & 2017 & Infant fecal metagenome & This study \\
\hline CDC317 & Candida & Parapsilosis & 13030174 & 9 & 2091826 & 93 & NA & Clinical skin isolate & $\begin{array}{l}\text { Butler et al. } 2009 \\
{[22]}\end{array}$ \\
\hline GA1 & Candida & Parapsilosis & 13025060 & 39 & 1114083 & 93 & NA & Clinical human blood isolate & $\begin{array}{l}\text { Pryszcz et al. } \\
2013 \text { [23] }\end{array}$ \\
\hline CBS1984 & Candida & Parapsilosis & 13044404 & 25 & 962200 & 92 & NA & Olive fruit isolate & $\begin{array}{l}\text { Pryszcz et al. } \\
2013 \text { [23] }\end{array}$ \\
\hline CBS6318 & Candida & Parapsilosis & 13050515 & 28 & 1691491 & 93 & NA & Healthy skin isolate & $\begin{array}{l}\text { Pryszcz et al. } \\
2013 \text { [23] }\end{array}$ \\
\hline N1_023 & Candida & Albicans & 13456346 & 1675 & 15180 & 94 & 2012 & Infant fecal metagenome & This study \\
\hline N2_070 & Candida & Albicans & 13540857 & 1614 & 14761 & 93 & 2012 & Infant fecal metagenome & This study \\
\hline N5_264 & Candida & Albicans & 11647081 & 746 & 27434 & 85 & 2015 & Infant fecal metagenome & This study \\
\hline S3_003 & Candida & Albicans & 11972257 & 1049 & 14710 & 87 & 2017 & Infant mouth metagenome & This study \\
\hline S3_016 & Candida & Albicans & 10068784 & 802 & 19749 & 86 & 2018 & $\begin{array}{l}\text { Infant mouth, skin, and gut } \\
\text { metagenome coassembly }\end{array}$ & This study \\
\hline SP_CRL & Candida & Albicans & 12561678 & 897 & 22840 & 91 & NA & Infant fecal metagenome & $\begin{array}{l}\text { Olm et al. } 2019 \\
{[18,21]}\end{array}$ \\
\hline
\end{tabular}


were named after their sample of origin. For comparison to isolate genomes, we analyzed 4 previously published C. parapsilosis and 51 C. albicans isolate genomes.

\section{Candida genomic variability}

To characterize genomic variability in the strains of $C$. albicans and $C$. parapsilosis represented by metagenome-derived genomes, we identified singlenucleotide variants (SNVs) by mapping reads against completed reference genomes (strain SC5314 for C. albicans and CDC317 for C. parapsilosis). C. albicans genomes ranged from 3.2 to 9.9 heterozygous SNVs per kb (heterozygosity), whereas C. parapsilosis genomes ranged from 0.12 to 0.38 heterozygous SNVs per kb. Heterozygous SNVs were defined as SNVs with two or more alleles detected in a single sample, indicating different alleles between chromosomes. Thus, we infer that, compared to C. albicans, C. parapsilosis displays very low genetic variability between its diploid chromosome pair, which can be indicative of low genetic variability in the hospital environment and primarily asexual reproduction [24].

Low heterozygosity in C. parapsilosis genomes has been reported for previously sequenced genomes [23].
Interestingly, C. parapsilosis genomes derived from our fecal metagenomes showed even lower overall heterozygosity than pure culture reference genomes (Figure S1). In general, this would not be expected because withinsample population diversity due to sampling of a microbial community should inflate measures of genomic heterozygosity. Thus, the lower genomic heterozygosity may be reflective of infants being initially colonized by essentially a single $C$. parapsilosis genotype.

Because multiple new strains were sequenced from the same hospital, the phylogenetic relationships of new and previously sequenced strains from the same hospital were of interest from the perspectives of the persistence of Candida populations in the hospital environment and transfer from room to human. To place the hospital and gut-associated sequences in context, we first compared those genomes to available reference genomes from NCBI using pair-wise average nucleotide identity (ANI) and by construction of single nucleotide variant (SNV) trees (Fig. 1A, Figure S1-S2). L2_023 was not included due to low sequencing coverage. $C$. albicans strains were spread throughout the tree of known C. albicans diversity (Figure S2) whereas C. parapsilosis strains from infant gut and NICU samples were clustered on a single

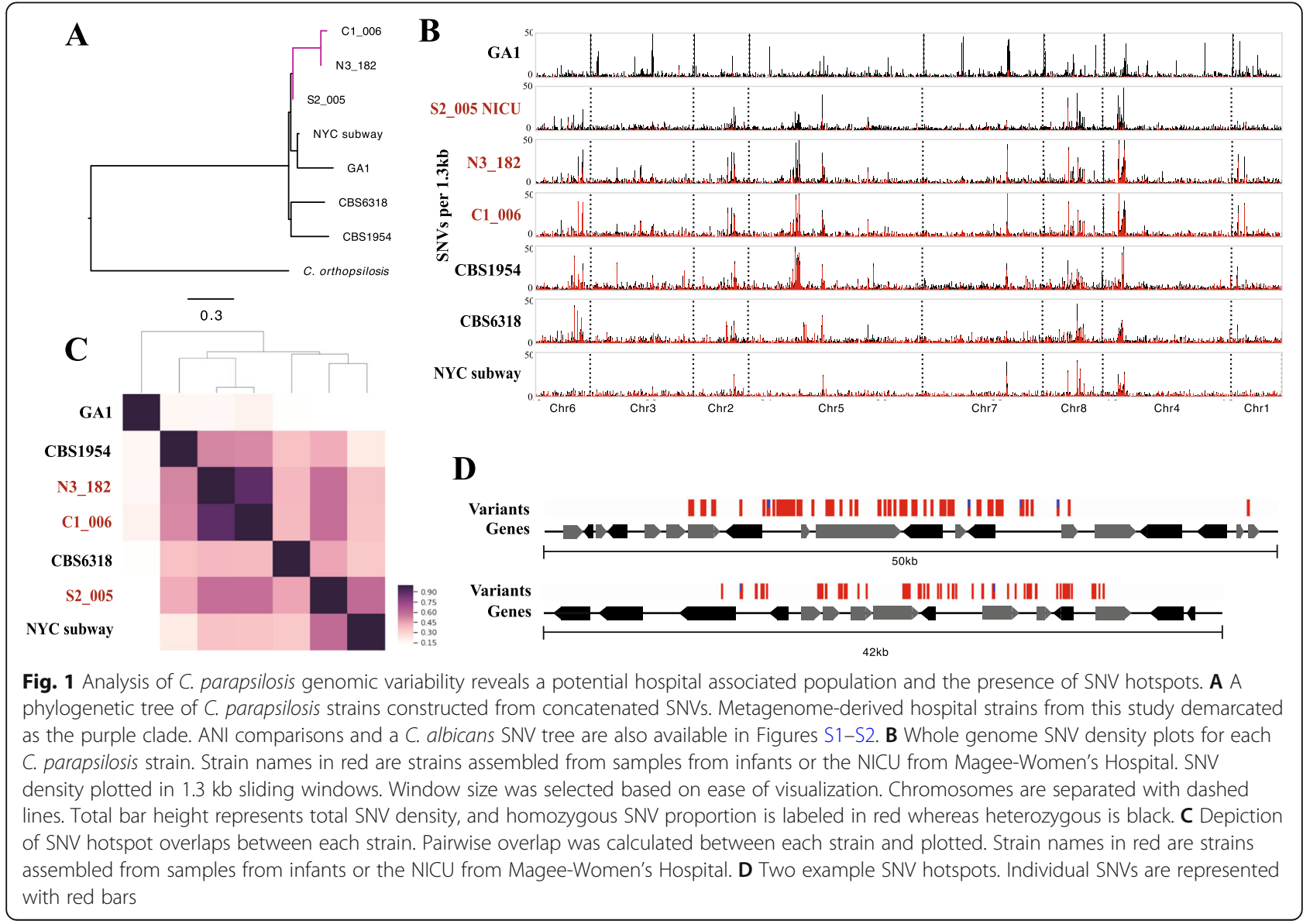


branch (Fig. 1A) separate from other reference hospital and environmental strains. Further, the two infant gut strains, sampled years apart (Table 1), were nearly identical (99.99\% identity). We verified this with whole genome alignments of the hospital and gut sequences (Figure S1-S2). We thus infer that the hospital room and gut C. parapsilosis strains are very closely related and are indicative of a possible hospital-associated $C$. parapsilosis strain sequenced multiple times, years apart.

Based on analysis of population structure of all seven unique C. parapsilosis genomes (Figure S3), we predicted six distinct $C$. parapsilosis ancestral populations. The exception is the fecal strain N3_182, which appears to be a recombinant admixture of the ancestral populations NICU strain S2_005 and the fecal strain C1_006. Given that N3_182 was sequenced 4 years before C1 006 (Table 1), both parental strains may have existed in the hospital environment prior to hybridization. The findings provide further evidence of distinct hospitalassociated C. parapsilosis strains, a hybrid of which colonized a premature infant. However, it may be difficult to accurately determine fine-grained population structure with small genome sample sizes, and future sequencing of $C$. parapsilosis genomes may further clarify this result.

\section{C. parapsilosis SNV hotspots as indicators of genes under selection}

To investigate whether genomes sampled from the hospital could provide evidence of evolutionary adaptation to this environment, we visualized the spatial distribution of C. parapsilosis genomic diversity in the newly reconstructed genomes by mapping reads from each genome to a reference sequence (CDC317, recovered from a clinical sample) and calling SNVs. We plotted the density of SNVs in $1.3 \mathrm{kbp}$ sliding windows across the genome of each strain (Fig. 1B). Both heterozygous and homozygous SNVs are largely evenly distributed throughout the genome, with the exception of a few small regions with highly elevated SNV counts (regions of elevated diversity) that we refer to as SNV hotspots (Fig. 1B).

Interestingly, SNV hotspots show a high level of conservation between all strains (Fig. 1C). The one exception is reference strain GA1 cultured from human blood [23], which shares only $~ 10 \%$ of its SNV hotspots with any other given strain. Notably, the NYC subway strain is fairly similar to the clinical reference strain CDC317 used for mapping (few and minor hotspots) whereas our hospital sequences share SNV hotspots with environmental reference strains CBS1954 and CBS6318 (one isolated from an olive and the other from healthy human skin).
To provide a more complete view of SNV hotspots and ensure they were not an artifact of SNV hotspots solely present in the CDC317 reference genome, we also mapped the reads from each population to three other genomes (environmental strains CBS1984 and CBS6318, and the GA1 blood isolate, Figure S4). The number of SNV hotspots ranged from 16 to 45 , and the regions were $5 \mathrm{~kb}$ to $24.5 \mathrm{~kb}$ in length. Due to the large size of the SNV hotspots, each hotspot overlaps a number of individual genes with SNVs spread both within and between genes (Fig. 1D). In total, 376 genes are present within a SNV hotspot in at least one strain. No particular KEGG family or PFAM domain was significantly enriched in SNV hotspots.

\section{Multicopy RTA3 gene}

Another explanation for SNV hotspots could be due to gene copy number variation, as recent duplications of a region acquire mutations yet reads from these duplications map back to a single location. Overall, when windowed genomic coverage is plotted alongside SNV density (Fig. 2A), this is clearly not the case. However, across the entire genome two regions of high coverage (Fig. 2A), indicating high copy number variation, were identified and neither correspond to SNV hotspots. The first high copy number region contains an estimated 1728 copies of the $18 \mathrm{~S}, 25 \mathrm{~S}, 5 \mathrm{~S}$, and 5.5S rRNA genes (Table S1, Fig. 2B). The variation in rRNA copy number may indicate a range of maximum growth rates [25]. The second region, which corresponds to the lipid translocase RTA3 gene and flanking sequence, is present in 5-16 copies (Table S1) in strains C1_006, N3_182, L2 023, S2_005, and NYC_subway but is single-copy in the four isolate genomes (Fig. 2B). Interestingly, RTA3 has been implicated in resistance to azole class antifungal drugs such as fluconazole in C. albicans [19]. The high copy number RTA3 genes also have no detectable SNVs and different boundaries in each strain, suggesting the duplications may be recent and independent events in each strain.

\section{In situ metatranscriptomics and metaproteomics}

Given most work with Candida species is performed in pure culture or in murine models, little is known about their behavior in the human gut. We hypothesized performing metatranscriptomics and metaproteomics on infant fecal samples with Candida would reveal unique transcriptomic and proteomic profiles, indicative of differences in metabolism and behavior between culture and in situ settings. Two candidate infants were identified: infant 06 with a documented Candida blood infection (Fig. 3) and infant 74 with a documented Candida lung infection. Both infants were treated with fluconazole shortly after detection of Candida infection (Fig. 3, 


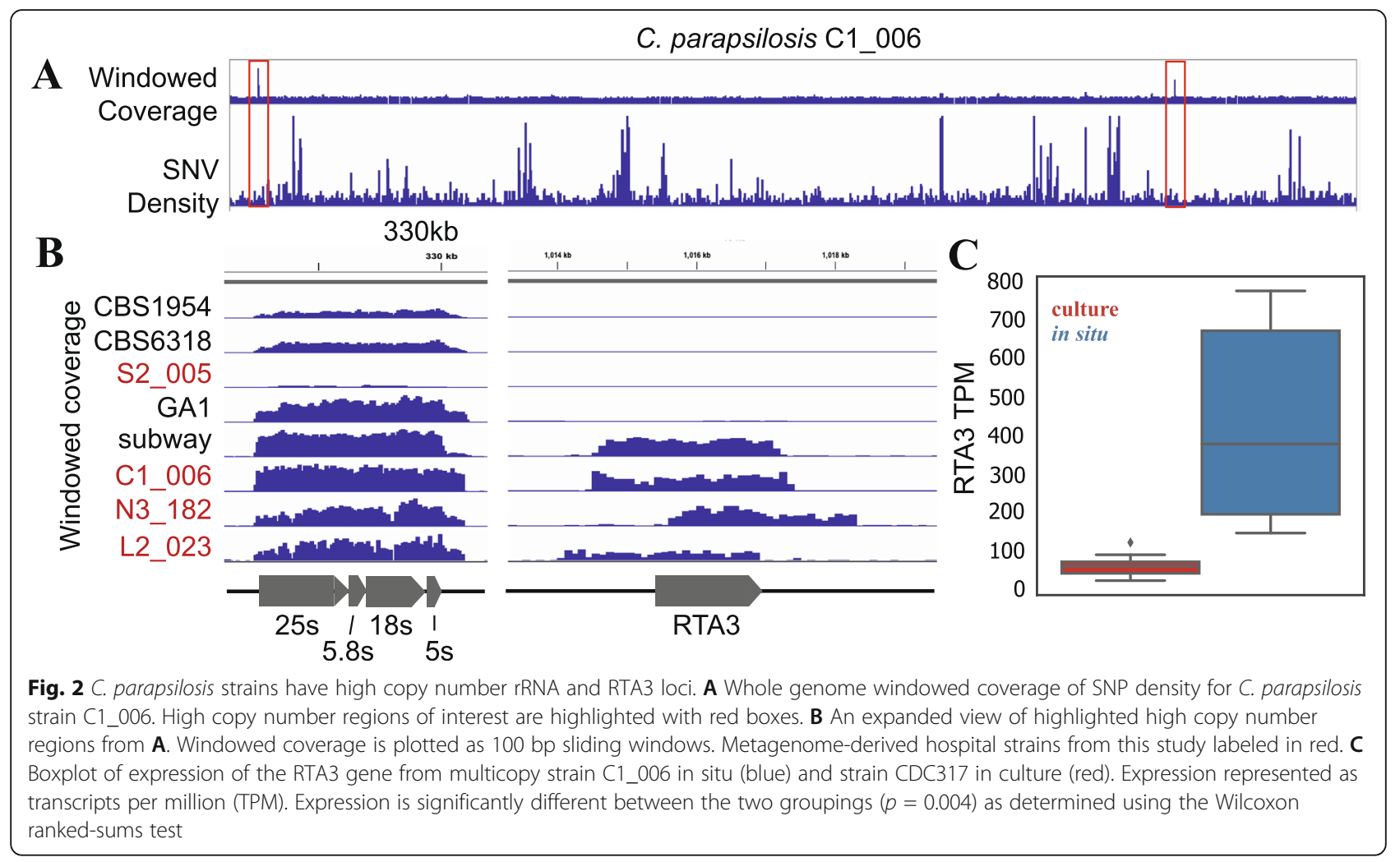

Table S2). Metagenomic, metatranscriptomic, and metaproteomic datasets were generated from fecal samples at five to six timepoints for each infant. In infant 74, no Candida species were detected in the generated datasets (Figure S4). However, in infant 06, metagenomic sequencing confirmed the presence of C. parapsilosis (strain C1_006) in the fecal samples. De novo gene prediction was performed on the metagenome-derived C. parapsilosis genome and the resulting gene models were used for mapping transcriptomic reads and proteomic peptides (Fig. 3).
In addition to $C$. parapsilosis, genomes were recovered for three bacterial species in infant 06: Enterococcus faecalis, Lactobacillus gasseri, and Staphylococcus epidermidis. It is not uncommon for only four organisms, or even fewer, to be present within a premature infant gut metagenome as the infant gut is normally sterile at birth and premature infants in particular typically receive antibiotics in the first weeks of life [21]. Interestingly, in every infant where a Candida genome was assembled or detected through read mapping, E. faecalis was also present $(N=7)$. C. parapsilosis is highly abundant

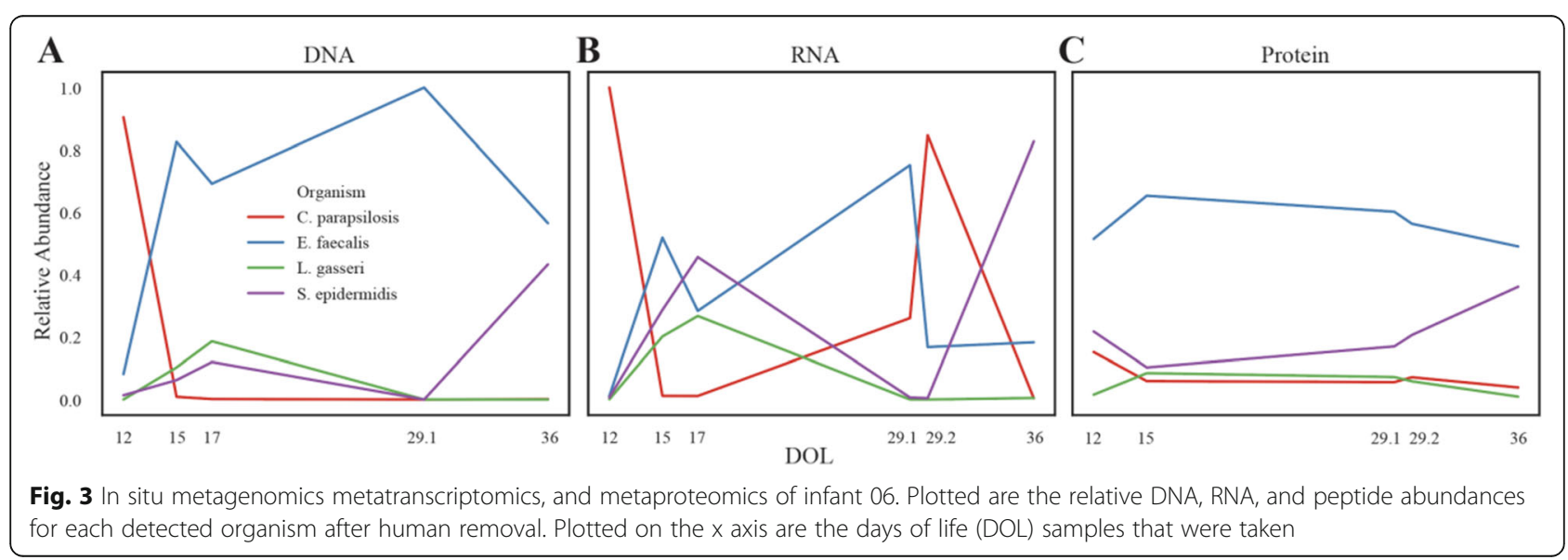


relative to other organisms in the first 20 days of life before quickly being replaced or outnumbered, largely by E. faecalis. Similar abundance patterns have been observed previously for microbial eukaryotes in neonatal fecal samples [18]. C. parapsilosis transcriptomic abundance shows a similar pattern to the DNA abundance but transcription remains detectable at later time points (Fig. 3). In contrast, C. parapsilosis proteomic abundance remained relatively stable over all timepoints.

\section{C. parapsilosis expression in situ vs. culture settings}

Given most work with $C$. parapsilosis has been performed on pure cultures, we wondered if there are differences in behavior and metabolism in situ that would be detectable by comparing transcriptomic datasets. For comparison, C. parapsilosis strain C1_06 was isolated from infant 06 fecal material on DOL 12. Transcriptomic datasets were then generated for cultures of the
C1_06 isolate grown in YPD at $30{ }^{\circ} \mathrm{C}$ to replicate standard Candida isolate culture conditions. In addition, we downloaded raw sequencing reads from publicly available C. parapsilosis RNAseq experiments [23, 26], including datasets from multiple strains (CDC317, CBS1954, and CBS6318) and varying culture conditions, including different media, growth temperatures, and oxygen concentrations. A hierarchical clustering of expression of CDC317 transcripts reveals a clearly distinct transcriptomic profile between in situ and all culture settings (Fig. 4A). Importantly, C1_06 culture transcriptomes cluster closer to culture transcriptomes of various other strains than to C1_06 in situ samples. Notably, in situ samples are also extremely variable; clustering as far apart from one another as from the culture samples (Fig. $4 \mathrm{~A})$. We quantitatively identified differentially expressed transcripts between culture and in situ settings with DESeq2 and found that $53 \%$ of transcripts were

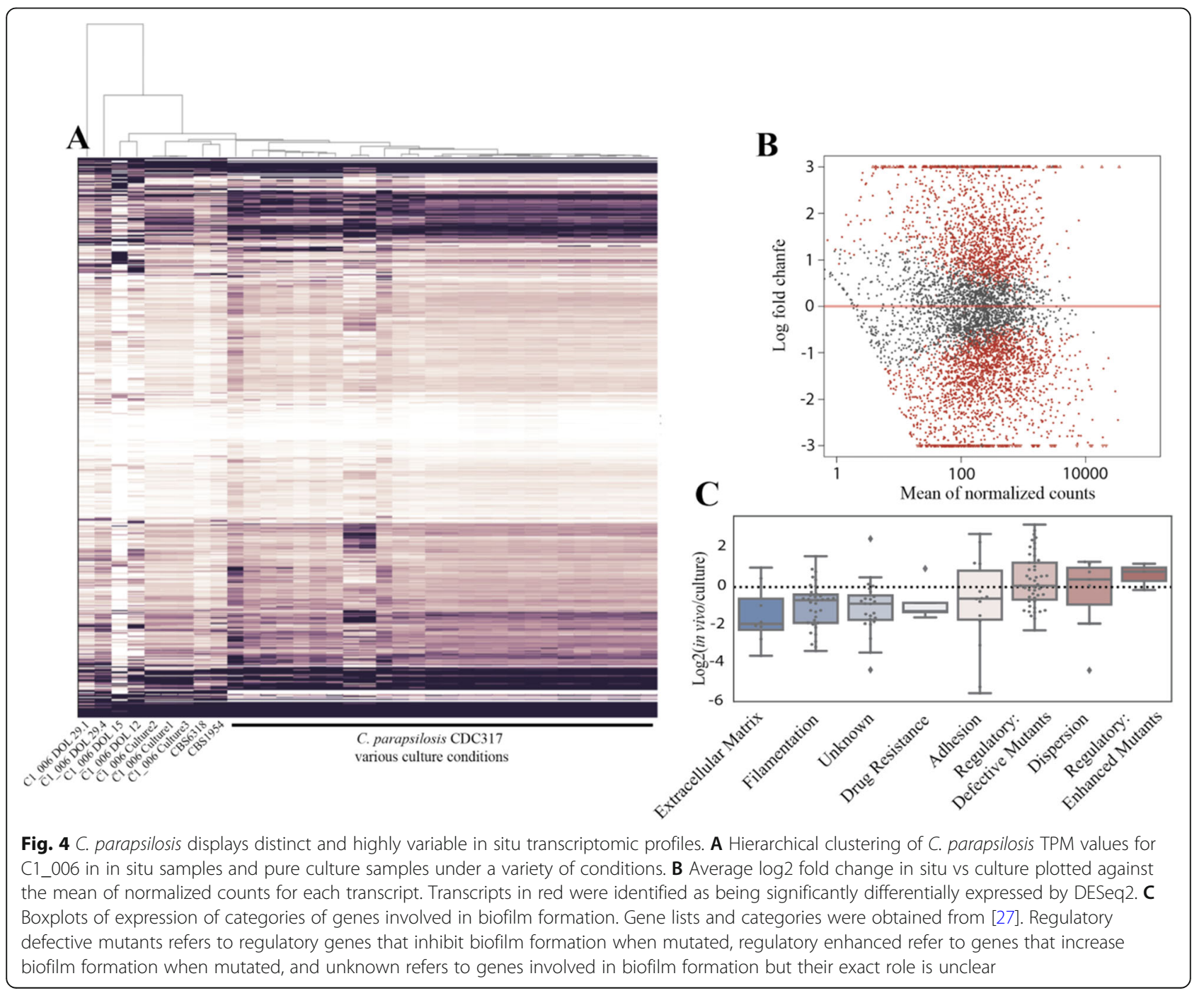


significantly differentially expressed; $23 \%$ up in situ, $30 \%$ down (Fig. 4B), further highlighting the stark differences between in situ and culture settings.

Biofilm formation is an important virulence factor for Candida species, often contributing to the development of systemic infections [27, 28]. We were interested in whether the expression of virulence factors was enriched in situ, given the samples were obtained from an infant with a documented Candida blood infection. We obtained a list of well-characterized biofilm formation genes from C. albicans [27], identified orthologs in $C$. parapsilosis and compared their expression in situ to culture settings. Biofilm formation showed lower expression overall in situ (Fig. 4C).

In situ and culture transcriptome samples were differentiable in a principal component analysis (PCA), paralleling the hierarchical clustering of $C$. parapsilosis transcriptomes (Fig. 5A), although C1_006 culture transcriptomes did not cluster as closely to other strain culture samples in this analysis. We performed a sparse partial least squares discriminant analysis (sPLS-DA), treating each transcript as a variable, to try and identify important features able to discriminate between in situ and culture in a multivariate space (Fig. 5B, Figure S5, Table S3). Important features were enriched for mitochondrial and aerobic respiration genes (9/50) and uncharacterized genes $(11 / 50)$.

We were curious to see if the multicopy RTA3 gene in infant strain C1_006 (Fig. 2B) showed increased expression as compared to the single copy RTA3 gene in reference strain CDC317. Indeed, the expression of the RTA3 in strain C1_006 is significantly higher $(p=0.004$,
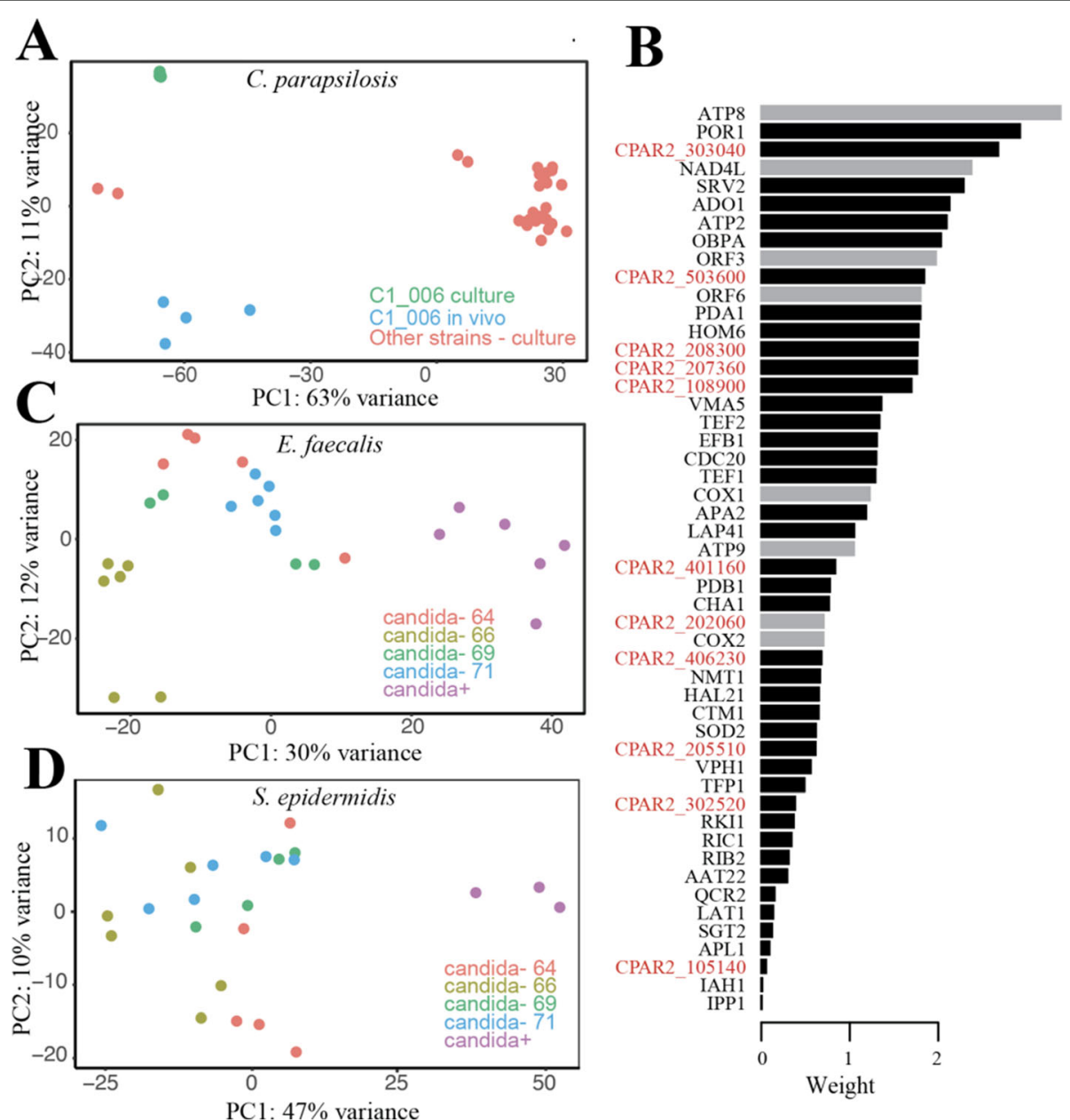

Fig. 5 Presence of C. parapsilosis affects bacterial community member's expression. A PCA of C. parapsilosis in situ and pure culture transcriptomes. B Depiction of features identified by SPLS-DA for separating C. parapsilosis in situ and pure culture transcriptomes. Plotted are the feature weights. Black bars are genes that exhibited higher expression on average in situ whereas grey had higher average expression in culture. Genes labeled in red correspond to proteins of unknown function. C, D PCAs of E faecalis (C) and S. epidermidis (D) transcriptomes from infant microbiomes both with and without detected C. parapsilosis. Candida-negative transcriptomes were from four different infants (published previously; Sher et al. 2020) denoted as 64, 66, 69, and 71 
Fig. 2C), suggesting a role of this gene duplication as a way to increase overall expression of RTA3. Interestingly, there was no significant difference in strain $\mathrm{C}_{-}$ 006 RTA3 expression between culture and in situ settings (Figure S6A), and we did not see an increase in expression following fluconazole treatment in situ (Figure S6B), indicating RTA3 expression may be constitutively higher in C1_006 regardless of condition. However, it is worth noting we were unable to obtain samples until 7 days after fluconazole treatment and any treatment effect on expression may have already passed.

\section{C. parapsilosis impact on bacterial expression}

E. faecalis, S. epidermidis, and L. gasseri bacteria in infant 06 had transcripts sequenced at high depths at multiple time points (Fig. 3). So, it was possible to investigate whether the presence or absence of $C$. parapsilosis had a distinguishable effect on their transcriptomic profiles. We compared bacterial transcription in these samples to transcription patterns of bacteria in the absence of Candida using previously reported datasets (21 samples for E. faecalis and 20 samples for S. epidermidis [29]). The analysis was not possible for L. gasseri as this bacterium was not present in any of the metatranscriptomes used for comparison. The transcriptomes of $S$. epidermidis were distinguishable between the presence and absence of $C$. parapsilosis, and this effect appears to be independent of infant of origin and thus the bacterial strain variant type (Fig. 5C, D). The effect is also present for E. faecalis, although less clear and could possibly be explained by variance across infants. This result suggests $C$. parapsilosis has a large impact on the behavior and metabolism of other gut community members. In addition, the expression of $E$. faecalis genes previously shown to negatively impact $C$. albicans virulence [15] showed no significant difference in expression between $C$. parapsilosis negative and positive samples.

Important features identified from a sPLS-DA on Candida-positive vs. Candida-negative samples included a subset of E. faecalis ribosomal proteins (Table S3, Figure S5). Additionally, ribosomal proteins all showed higher expression in situ, suggesting increased E. faecalis growth rate in the presence of $C$. parapsilosis. Other important features included mannitol-specific phosphotransferase system (PTS) transporters upregulated in Candida-positive samples and downregulated mannosespecific PTS transporters (Table S3). Furthermore, Mannitol-1-phosphate 5-dehydrogenase, an enzyme responsible for the conversion of D-mannitol to fructose, was upregulated in Candida-positive samples, indicating an increased capacity for degradation of mannitol in addition to import (Table S3). Important features in $S$. epidermidis were less clear, but again included a subset of ribosomal proteins as well as beta-lactamases, both with increased expression in situ (Table S3).

\section{Transcriptomics enriched gene functions}

Given the large differences in transcriptomes between culture and in situ C. parapsilosis, we looked for functions enriched in either setting (Fig. 6, Table S4). DESeq2 identified groups of differentially expressed genes that were too large to be informative, so more restrictive cutoffs were used. Up in situ was defined as having > $3 \log 2$ expression in situ whereas down in situ was defined as $<-3 \log 2$ expression in situ. Up in situ was enriched for KEGG families for LSM 2-8 and 1-7 complexes, a family of proteins involved in mRNA metabolism highly conserved in eukaryotes [30], as well as Cytochrome c oxidase and bc1 complex and proteins without an annotated KEGG family (Fig. 6, Table S4). Down in situ is enriched for helicase and polysaccharide synthase PFAM domains. Additionally, proteins without an annotated KEGG family (unknown function) were enriched in both groups (Table S4).

\section{Proteomics}

Across the 10 proteome samples from 5 timepoints in infant 06, 7063 protein groups (groups of similar sequences that cannot be distinguished because the peptides are shared) were quantified, with an average of 4872 protein groups at each time point. Among these were 5312 human and 1751 protein groups from $C$. parapsilosis and bacteria (Supplemental Figure S7). Human protein groups dominate (90\% of the peptide abundances in each sample) because the limited amount of fecal material precluded depletion of human cells before cellular lysis and protein extraction. Among the quantified human protein groups, 324 of the 480 involved in neutrophil degranulation (67\%) were identified, with an average of 294 protein groups detected at each sampling point. This indicates an active host immune response [31] (Supplemental Table S5).

While the high representation of human protein groups reduces the coverage depth of the microbial membership, it allows for simultaneous examination of both host and microbiome activities. We quantified 349 C. parapsilosis protein groups across all samples measured, with a minimum of 126 C. parapsilosis protein groups per sample. While this represents only $\sim 6 \%$ of the predicted $C$. parapsilosis proteome, the data enabled observation of stability across time and determination of some of the general metabolic activities of this organism (Supplemental Figure S8). We detected evidence for $C$. parapsilosis core metabolic activities such as glycolysis, the TCA cycle, and organic acid metabolism. Repeated detection of similar abundances of these proteins across 


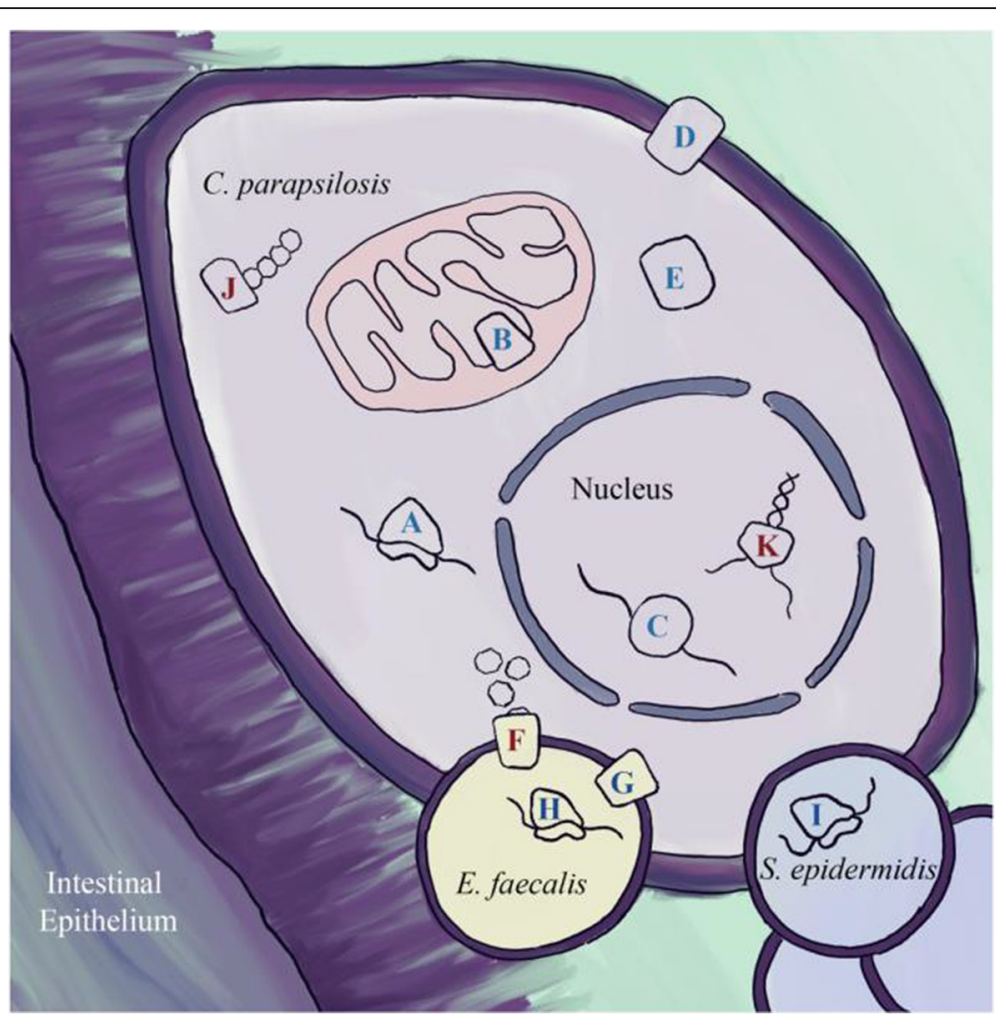

Fig. 6 In situ enriched gene categories. Diagram depicting C. parapsilosis in the context of the infant gut, highlighting gene categories or families that were significantly enriched in differentially expressed genes between in situ and culture. Blue letters represent functions with higher expression in situ, while red represent functions with lower expression in situ. See Table S5 for details. A Ribosomal proteins, B cytochrome C oxidase subunits, C LSM complexes, D proton antiporters, $\mathbf{F}$ E. faecalis mannose transporters, $\mathbf{G} E$. faecalis mannitol transporters, $\mathbf{H} E$. faecalis subset of ribosomal proteins, I S. epidermidis subset of ribosomal proteins, J C. parapsilosis polysaccharide synthases (downregulated in situ), K C. parapsilosis helicases (downregulated in situ).

the 24-day timespan of collected samples indicates the stability of C. parapsilosis in the gut environment.

The abundant, significantly enriched protein groups from $C$. parapsilosis were ribosomal and F-Type ATPase proteins (Fig. 6), HSP70 and proteins with actin PFAM domains (Table S4). Protein groups with the most peptide evidence were involved in protection from oxidative stress (e.g., superoxide dismutase). This suggests that $C$. parapsilosis was actively responding to, and adapting to, environmental stressors. Included in the set of highly abundant proteins were some encoded in genome regions within SNV hotspots. However, we found no significant association between protein abundance and association with these hotspots. We also examined the most abundant proteins in the bacterial species. In $E$. faecalis and S. epidermidis, proteins of the Lac pathway were among the most abundant bacterial proteins. This suggests that lactose may be an important substrate for these community members in situ.

\section{Discussion}

Fungal pathogens are known to have hospital reservoirs. For example, the water supply system of a pediatric institute was shown to be a reservoir for Fusarium solani [32]. A NICU outbreak of the fungi Malassezia pachydermatis was linked to the dog of a healthcare worker [33], although persistence via long-term carriage by a healthcare worker vs. continual passage between infants and rooms (or a combination of these) could not be resolved. However, much remains to be learned about where reservoirs of hospital-associated fungi are and how long strains persist in them. In contrast to previous studies of C. parapsilosis utilizing pure culture and model systems, we applied genome-resolved metagenomics, metatranscriptomics, and metaproteomics to study $C$. parapsilosis in the context of the infant gut and hospital rooms of a neonatal intensive care unit. We detected novel, near identical C. parapsilosis genomes sequenced years apart in separate infants, suggesting transmission of members of a fungal population from reservoir to infant or infant to reservoir to infant. It is worth noting that although the strains are near-identical, the multicopy RTA3 locus in each strain had different boundaries and different copy numbers. This observation suggests that these two strains are very closely related members of a somewhat more diverse hospital adapted population. 
Population genomic analyses of reconstructed genomes revealed multiple, independent instances of copy number gain of the RTA3 gene. RTA3, a lipid translocase, has been implicated in resistance to azole class antifungal drugs such as fluconazole in C. albicans [19]. The RTA3 gene is frequently overexpressed in resistant isolates and increased expression of RTA3 increases resistance to fluconazole whereas deletion of the RTA3 gene results in increased azole susceptibility [19]. Copy gain of this gene in C. parapsilosis strains may represent a mechanism for rapid adaptation to fluconazole, the most widely used antifungal in most hospitals [19], as a means by which to increase its expression and thus its resistance. Similar gene copy number gains have been reported for the human amylase gene, hypothesized to be in response to increases in starch consumption [34]. Indeed, RTA3 expression in situ from strain C1_006, which has RTA3 in multicopy, was significantly increased as compared to single copy strain CDC317 in culture [26] (Fig. 2C). The high likelihood that the copy number gain occurred independently in multiple strains suggests selection for this particular genomic feature. Identifying mechanisms of antifungal resistance is of particular importance given $3-5 \%$ of $C$. parapsilosis strains are already resistant to fluconazole [10] and our relative inability to deal with infections of drug-resistant fungi.

Examining the genomic distribution of SNVs within the genomes of each $C$. parapsilosis strain revealed the presence of SNV hotspots. Interestingly, no particular KEGG family or PFAM domain was significantly enriched within SNV hotspots. This, combined with the fact that SNVs within hotspots are spread both within and between genes, may be indicative of the identified SNV hotspots being recombination hotspots, or locations where many additional SNVs hitchhike along with SNVs under selection as many of these SNVs, particularly those in non-coding regions, are likely to have little to no effect on function.

Many of these SNV hotspots are shared between strains, some of which are specific to the hospital and infant gut strains. Unlike C. albicans, C. parapsilosis is not an obligate commensal of mammals [6]. Consequently, some regions of the $C$. parapsilosis genome may be under selection for adaptation to the hospital, in addition to the gut environment. Further supporting the idea that some genomic innovation is associated with adaptation to the built environment, the NICU strain clustered the most closely to the NYC subway strain based on SNV hotspot overlap (Fig. 1C). These two strains are geographically and phylogenetically distinct, but the shared regions of diversification may be related to their common need to adapt to the built environment.

Metatranscriptomics of infant fecal samples revealed C. parapsilosis transcriptomes that are both highly variable and distinct from those of culture samples. Interestingly, the degree of variance exhibited by transcriptomes of the same population in the same infant over a few day period was greater than that observed between C. albicans white and opaque phenotypes (Figure S9) [35]. The C. albicans white and opaque phenotypes differ in their appearance [36], mating style [37], and environmental conditions they are best adapted to [38, 39], and represent two exceptionally distinct Candida phenotypes. The high variability in $C$. parapsilosis is likely the result of changing conditions presented in the gut, including microbial community composition as well as the developing physiology of the host. Varying stages of infection and/or response to antifungal treatment may also have had an effect, but more dense time-series and additional infants would be required to elucidate these effects.

In contrast to the large changes in $C$. parapsilosis RNA and DNA relative abundances over time, C. parapsilosis peptide relative abundance remained stable over the study period. It is not uncommon to see different signals from transcripts and proteins [40], in part because proteins can persist for relatively long periods of time compared to transcripts. The most abundant proteins in the proteomics dataset have a HSP70 domain found in heat shock proteins (HSP). In C. albicans, HSP have been documented to help control virulence by interacting with regulatory systems and to enable drug resistance [41].

The presence of $C$. parapsilosis within infant gut samples may impact the transcriptomes of bacterial gut community members. Important features for separating Candida-positive and Candida-negative E. faecalis transcriptomic samples included a suite of upregulated mannitol transporters and downregulated mannose transporters (Table S3). C. parapsilosis strain SK26.001 is documented as producing mannitol [42] and mannose, in the form of the polysaccharide mannan which can be an important component of extracellular polysaccharides produced by Candida [43]. Interestingly, a characteristic of $E$. faecalis is its ability to grow by fermenting mannitol [44]. Given the potential for interaction between $E$. faecalis and C. parapsilosis, it is possible that the presence of $C$. parapsilosis induces a substrate switch in $E$. faecalis from mannose, an important component of $C$. parapsilosis biofilm matrix, to mannitol, a sugar produced by $C$. parapsilosis under some conditions.

Interestingly, statistical tests detected a subset of ribosomal proteins as important features for separating Candida-positive from Candida-negative samples for both E. faecalis and S. epidermidis (Table S3) based on transcription. In recent years, ribosomal heterogeneity, in which ribosomal protein subunits are swapped out or missing from individual ribosomes, has gained traction 
as a way for organisms to regulate translation [45-47]. Ribosomal heterogeneity may be being utilized as an additional regulatory measure to adapt to the rapidly changing gut microbial context. Alternatively, fluctuations in ribosomal subunit abundance could be to maintain ribosomal homeostasis [48], or individual ribosomal subunits could be performing functions unrelated to protein synthesis [49].

Biofilm formation is an important virulence factor of Candida infections [50]. Infant 06 had a documented Candida blood infection, and such infections are commonly systemic [51]. Interestingly, despite infection, Candida biofilm formation genes were relatively less expressed in situ in the gut of Infant 06 as compared to expression levels previously reported over a range of culture conditions. Similarly, genes with a PFAM domain for polysaccharide synthase, genes potentially important for the generation of Candida biofilm matrices [43], were less expressed in situ than in cultures. Thus, biofilm formation may not be an important component of every infection.

The prevalence of transcripts of uncharacterized genes in the in situ transcriptomes (Fig. 5B; Table S3) is particularly interesting. C. parapsilosis and other Candida species are rarely studied in a microbial community context, leaving gaps in understanding of genes required for organism-organism interactions. We suspect that some of the highly expressed genes are important for Candida interactions with bacteria and other community members. Thus, they represent important targets for future co-culture-based investigations.

A limitation of this study was obtaining fecal samples with sufficient Candida DNA, RNA, and protein for analysis. Consequently, although we present the first in situ metatranscriptomics and metaproteomics for C. parapsilosis, the data analyzed is for a single strain in a single infant. It is perhaps not unexpected that in situ expression patterns differed significantly from those observed in culture settings. However, metatransciptomes from more C. parapsilosis strains and more infants that are recovered under highly standardized conditions are needed to determine the contributing factors, such as the coexisting bacteria and infant gut conditions that lead to these differences. Development of methods to more reliably recover low abundance microbial eukaryotic material in the midst of the bacterially dominated gut will be crucial for further insights.

\section{Conclusions}

We applied genome-resolved metagenomics, metatranscriptomics, and metaproteomics to recover genomes for, and study the behavior of, C. parapsilosis in situ. We showed C. parapsilosis has a highly distinct transcriptomic profile in situ vs in culture. Further, the extreme variability in the in situ transcriptome data indicates the considerable effect the gut microbial community and human host may have on C. parapsilosis behavior and metabolism. Overall, these results demonstrate that in situ study of C. parapsilosis and other Candida species is not only possible but necessary for a more holistic understanding of their biology.

\section{Methods}

\section{Metagenomic sampling and sequencing}

All infant fecal metagenomes used in this study were derived from infants housed in the Magee-Womens Hospital (Pittsburgh, PA). This study made use of previously published infant datasets: NIH1 [52], NIH2 [53], NIH3 [54], NIH4 [55], Sloan2 [53], and SP_CRL [56], as well as several new datasets including multiple timepoints from infant 06 and infant 74, and samples L2_023, S3 003, and S3_016.

For newly generated metagenomic sequencing from infant 06 and infant 74, total genomic DNA and total RNA were extracted from previously unanalyzed fecal samples using Qiagen's AllPrep PowerFecal DNA/RNA kit (Qiagen) and subsequently split into DNA and RNA portions. The aliquot used for metagenomic sample preparation was treated with RNase A. DNA quality and concentration were verified with Qubit (Thermofisher) and Fragment Analyzer (Agilent). Illumina libraries with an average insert size of 300 bps were constructed from purified genomic DNA using the Nextera XT kit (Illumina) and sequenced on Illumina's NovaSeq platform in a paired end $140 \mathrm{bp}$ read configuration, resulting in at least 130 million paired end reads from each library.

NICU metagenomic sampling was described and published previously [53]. All samples were collected from the same NICU at UPMC Magee-Womens Hospital (Pittsburgh, PA). In order to generate enough DNA for metagenomic sequencing, DNA was collected from multiple sites in the NICU and combined into three separate pools for sequencing. Highly touched surfaces included samples originating from the isolette handrail, isolette knobs, nurses hands, in-room phone, chair armrest, computer mouse, computer monitor, and computer keyboard. Sink samples included samples from the bottom of the sink basin and drain. Counters and floors consisted of the room floor and surface of the isolette. See previous publication for details $[53,57]$.

\section{Eukaryotic genome binning and gene prediction}

For each sample, sequencing reads were assembled independently with IDBA-UD [58]. Additionally, for each infant, reads from every time point were concatenated together. A co-assembly was then performed on the pooled reads for each infant with IDBA-UD in order to assemble sequences from low abundance organisms. The 
Eukaryotic porton of each sample assembly was predicted with EukRep [17] and putative eukaryotic bins were generated by running CONCOCT [59] with default settings on the output of EukRep. To reduce computational load, resulting eukaryotic bins shorter than 2.5 mbp in length were not included in further analyses. GeneMark-ES [60] and AUGUSTUS [61] trained with BUSCO [62] were used to perform gene prediction on each bin using the MAKER [63] pipeline. In addition, a second homology-based gene prediction step was performed. Each bin was identified as either $C$. parapsilosis or C. albicans and reference gene sets from C. parapsilosis CDC317 and C. albicans SC5314 were used for homology evidence respectively in a second-pass gene prediction step with AUGUSTUS [61], as implemented in MAKER [63].

\section{Bacterial genome binning and gene prediction}

For infant 06 and infant 74 metagenomes, bacterial genes were predicted on whole metagenomes using Prodigal in metagenome mode (-p meta option; Hyatt et al. 2012). Predicted proteins were functionally and taxonomically annotated by searching against Uniprot (The UniProt Consortium 2017), KEGG (Kanehisa et al. 2016), and Uniref90 (Suzek et al. 2007) with USEARCH (UBLAST) (Edgar 2010). Taxonomy for scaffolds was then determined by taking the consensus of closest hits of each individual gene sequence on a contig and determining the winner by majority. Bacterial genomes were then manually binned with ggKbase (ggkbase.berkeley.edu) utilizing coverage, $\mathrm{GC}$, and taxonomic information.

\section{SNV calling and detection of SNV hotspots}

In order to call variants in each Candida genome, reads from the sample in which a particular genome was binned from, or the publically available reads from SRA, were mapped back to the de novo assembled genome using Bowtie 2 [64] with default parameters. The PicardTool (http://broadinstitute.github.io/picard/) functions "SortSam" and "MarkDuplicates" were used to sort the resulting sam file and remove duplicate reads. FreeBayes (Garrison et al. 2012) was used to perform variant calling with the options "--pooled-continuous -F 0.01 -C 1." Variants were filtered downstream to include only those with support of at least $10 \%$ of total mapped reads in order to avoid false positives. SNV read counts were calculated using the "AO" and "RO" fields in the FreeBayes vcf output file.

SNV density was visualized across the CDC317 reference genome using a custom python script (https:// github.com/patrickwest/c_parapsilosis_analysis). SNV hotspots were quantitatively defined with $5 \mathrm{kbp}$ windows with a slide of $500 \mathrm{bp}$ across the genome, flagging windows with a SNV density at least three standard deviations above the genomic average SNV density, and merging overlapping flagged windows. Genes located within SNV hotspots as well as overlapping SNV hotspots between strains were identified with intersectBed [65].

\section{Candida phylogenetics and population structure}

For generation of a SNP tree for both C. parapsilosis and C. albicans, all publically available genomic sequencing reads for both species were downloaded from NCBI's short read archive (SRA), including 4 isolate C. parapsilosis read sets and 51 C. albicans sets. SNVs were called for each isolate read set using the same pipeline used for metagenome-derived genomes, as described above. A SNP tree was generated for $C$. parapsilosis and $C$. albicans using SNPhylo [66] with settings '-r -M 0.5 -1 2' and '-r -M 0.5 -l 0.8 ' respectively and visualized using FigTree (https:/github.com/rambaut/figtree/). For genomic average nucleotide identity (ANI) comparisons, 4 C. parapsilosis and 51 C. albicans reference genomes were downloaded from NCBI. Subsequently, dRep [67] in the 'compare_wf' setting was used to generate ANI comparisons for each genome. For inferring C. parapsilosis population structure, FreeBayes vcf files were converted to PLINK bed format with PLINK [68] and used as input for ADMIXTURE [69]. A total of $3785 \mathrm{SNVs}$ were used to infer ancestral populations. The predicted number of ancestral populations, K, was selected using ADMIXTURE's cross-validation procedure for values $1-8$.

\section{Detection of copy number variation}

Genomic copy number variation within the C. parapsilosis strains was searched for by mapping reads from the sample the genome was derived from to the C. parapsilosis CDC317 reference genome. Windowed coverage was then calculated across the genome in $100 \mathrm{bp}$ sliding windows using pipeCoverage (https://github.com/ $\mathrm{MrOlm} /$ pipeCoverage) and visualized with Integrated Genomics Viewer (IGV) [70]. Copy numbers for multicopy regions were estimated by dividing the average coverage of the windows located within the multicopy region by the average genomic coverage.

\section{Transcriptomic sequencing and analysis}

For in vivo metatranscriptome generation, total RNA was extracted from fecal samples using the AllPrep PowerFecal DNA/RNA kit (Qiagen) and subsequently treated with DNase. Purified RNA quality and concentration were measured using the Fragment Analyzer (Agilent). Illumina sequencing libraries were constructed with the ScriptSeq Complete Gold Kit (Illumina) without performing the rRNA removal step, resulting in library molecules with an average insert size of $150 \mathrm{bp}$. Sequencing was performed on Illumina's NextSeq platform in a 
paired end $75 \mathrm{bp}$ configuration, resulting in an average of 54 million paired end reads per sample.

For culture C. parapsilosis strain C1_06 transcriptomes, strain C1_06 was isolated from the stool of Infant patient 06 on day 12 of life. Cultures of this isolate were grown in YPD at $30{ }^{\circ} \mathrm{C}$ to mid-log phase. All cultures were pelleted, washed, and flash frozen in liquid nitrogen. RNA was extracted using the RiboPure RNA Purification kit (Ambion) and RNA samples were submitted to the JP Sulzberger Columbia Genome Center for library preparation and sequencing. Libraries were constructed using the Illumina TruSeq RNA library prep kit v2 and $100 \mathrm{bp}$ single-end reads were sequenced using the Illumina NovaSeq.

Transcriptomic reads from studies [23, 26] were downloaded from the SRA. Transcriptomic reads from each dataset were then mapped to $C$. parapsilosis reference strain CDC317 gene models with Kallisto [71] and transcript per million (TPM) values were used to compare expression levels across samples. Differentially expressed transcripts were identified using raw read counts with the R package DESeq2 [72]. Rlog transformation was applied to transcript read counts from each sample prior to generation of transcriptome PCAs. PCA plots were generated with DESeq2. Important features for separating C. parapsilosis in situ and culture as well as E. faecalis and S. epidermidis Candida-positive and Candida-negative samples were identified through the use of a sparse Partial Least Squares Discriminant Analysis (sPLS-DA) as implemented in the MixOmics package [73] on rlog transformed transcript read counts. MixOmics cross-validation (tune.splsda) was used with settings fold $=3$ and nrepeat $=50$ to estimate the optimal number of components (features) for separating each pair of sample types.

Genes were annotated with KEGG KOs and PFAM domains using HMMER with KOfam [74] and Pfam-A [75] HMM databases. Subsets of genes of interest (described in results) were then searched for significantly enriched KEGG families or PFAM domains with a hypergeometric distribution test as part of the R 'stats' package [76].

\section{Generation of proteomic datasets}

Lysates were prepared from $\sim 50 \mathrm{mg}$ of fecal material by bead beating in SDS buffer (4\% SDS, $100 \mathrm{mM}$ Tris- $\mathrm{HCl}$, $\mathrm{pH}$ 8.0) using $0.15-\mathrm{mm}$ diameter zirconium oxide beads. Cell debris was cleared by centrifugation $(21,000 \times g$ for $10 \mathrm{~min}$ ). Pre-cleared protein lysates were adjusted to 25 $\mathrm{mM}$ dithiothreitol and incubated at $85{ }^{\circ} \mathrm{C}$ for $10 \mathrm{~min}$ to further denature proteins and to reduce disulfide bonds. Cysteine residues were alkylated with $75 \mathrm{mM}$ iodoacetamide, followed by a 20-min incubation at room temperature in the dark. After incubation, proteins were isolated by chloroform-methanol extraction. Protein pellets were washed with methanol, air-dried, and resolubilized in $4 \%$ sodium deoxycholate (SDC) in $100 \mathrm{mM}$ ammonium bicarbonate $(\mathrm{ABC})$ buffer, $\mathrm{pH}$ 8.0. Protein samples were quantified by BCA assay (Pierce) and transferred to a 10-kDa MWCO spin filter (Vivaspin 500; Sartorius) before centrifugation at $12,000 \times g$ to collect denatured and reduced proteins atop the filter membrane. The concentrated proteins were washed with 100 $\mathrm{mM}$ ABC ( $2 \times$ the initial sample volume) followed by centrifugation. Proteins were resuspended in a $1 \times$ volume of $A B C$ before proteolytic digestion. Protein samples were digested in situ using sequencing-grade trypsin (G-Biosciences) at a 1:75 (wt/wt) ratio and incubated at $37{ }^{\circ} \mathrm{C}$ overnight. Samples were diluted with a $1 \times$ volume of $100 \mathrm{mM} \mathrm{ABC}$, supplied with another 1:75 (wt/wt) aliquot of trypsin, and incubated at $37{ }^{\circ} \mathrm{C}$ for an additional $3 \mathrm{~h}$. Tryptic peptides were then spin-filtered through the MWCO membrane and acidified to $1 \%$ formic acid to precipitate the residual SDC. The SDC precipitate was removed from the peptide solution with water-saturated ethyl acetate extraction. Samples were concentrated via SpeedVac (Thermo Fisher), and peptides were quantified by BCA assay (Pierce) before LC-MS/MS analysis.

Twelve micrograms of each peptide sample was analyzed by automated 2D LC-MS/MS using a Vanquish UHPLC with autosampler plumbed directly in-line with a Q Exactive Plus mass spectrometer (Thermo Scientific). A 100- $\mu \mathrm{m}$ inner diameter (ID) triphasic back column [RP-SCX-RP; reversed-phase $(5 \mu \mathrm{m}$ Kinetex C18) and strong-cation exchange ( $5 \mu \mathrm{m}$ Luna SCX) chromatographic resins; Phenomenex] was coupled to an inhouse pulled, $75 \mu \mathrm{m}$ ID nanospray emitter packed with $30 \mathrm{~cm}$ Kinetex $\mathrm{C} 18$ resin. Peptides were autoloaded, desalted, separated, and analyzed across four successive salt cuts of ammonium acetate $(35,50,100$, and 500 $\mathrm{mM}$ ), each followed by a 105-min organic gradient. Mass spectra were acquired in a data-dependent mode with the following parameters: a mass range of 400 to $1500 \mathrm{~m} / \mathrm{z}$; MS and MS/MS resolution of $35 \mathrm{~K}$ and 17.5 $\mathrm{K}$, respectively; isolation window $=2.2 \mathrm{~m} / \mathrm{z}$ with a 0.5 $\mathrm{m} / \mathrm{z}$ isolation offset; unassigned charges and charge states of $+1,+5,+6,+7$, and +8 were excluded; dynamic exclusion was enabled with a mass exclusion window of $10 \mathrm{ppm}$ and an exclusion duration of $45 \mathrm{~s}$.

MS/MS spectra were searched against custom-built databases composed of the concatenated sequenced metagenome-derived predicted proteomes from all timepoints, the human reference proteome from UniProt, common protein contaminants, and reversed-decoy sequences using Proteome Discover 2.2 (Thermo Scientific), employing the CharmeRT workflow [77]. Peptide spectrum matches (PSMs) were required to be fully tryptic with two miscleavages, a static modification of 
57.0214 Da on cysteine (carbamidomethylated) residues, and a dynamic modification of $15.9949 \mathrm{Da}$ on methionine (oxidized) residues. False-discovery rates (FDRs), as assessed by matches to decoy sequences, were initially controlled at $1 \%$ at the peptide level. To alleviate the ambiguity associated with shared peptides, proteins were clustered into protein groups by $100 \%$ identity for microbial proteins and $90 \%$ amino acid sequence identity for human proteins using USEARCH [78]. FDRcontrolled peptides were then quantified according to the chromatographic area under the curve (AUC) and mapped to their respective proteins. Peptide intensities were summed to estimate protein-level abundance based on peptides that uniquely mapped to one protein group. Protein abundance distributions were then normalized across samples using InfernoRDN [79], and missing values were imputed to simulate the mass spectrometer's limit of detection using Perseus [80] as annotated in the Reactome database [81].

\section{Supplementary Information}

The online version contains supplementary material available at https://doi. org/10.1186/s40168-021-01085-y.

\section{Additional file 1. \\ Additional file 2. \\ Additional file 3. \\ Additional file 4. \\ Additional file 5. \\ Additional file 6. \\ Additional file 7. \\ Additional file 8. \\ Additional file 9. \\ Additional file 10. \\ Additional file 11. \\ Additional file 12. \\ Additional file 13. \\ Additional file 14.}

\section{Acknowledgements}

We thank Spencer Diamond and Alex Crits-Christoph for their helpful discussions and advice on statistical methods as well as Michelle Tan, Rene Sit, and Norma Neff from Chan Zuckerberg Biohub Genomics Platform for providing sequencing resources.

\section{Authors' contributions}

PTW performed data analyses, prepared figures, and wrote the manuscript. SLP prepared figures, assisted in data analyses, and generated datasets. MRO and $\mathrm{YCL}$ assisted in data analyses. FBY, HG, BAF, and RB generated additional datasets. ADJ, MJM, and RLH provided guidance and assisted in data analyses. JFB provided guidance, assisted in data analyses, and wrote the manuscript. The authors read, approved, and contributed to the manuscript.

\section{Funding}

This research was supported by the National Institutes of Health $(\mathrm{NIH})$ under award RAI092531A, the Alfred P. Sloan Foundation under grant APSF-201210-05, and National Science Foundation Graduate Research Fellowships to P.W. under Grant No. DGE 1106400. This work used the Vincent J. Coates
Genomics Sequencing Laboratory at UC Berkeley, supported by NIH S10 OD018174 Instrumentation Grant.

\section{Availability of data and materials}

The datasets supporting the conclusions of this article are available in the NCBI BioProject repository, PRJNA471744 https://www.ncbi.nlm.nih.gov/ bioproject/?term=PRJNA471744, the Short Read Archive (SRA) SRR5420274 to SRR5420297, https://www.ncbi.nlm.nih.gov/sra/?term=SRR5420274, and https://github.com/patrickwest/c_parapsilosis_analysis. Candida genomes and sequencing reads from infant 06 are available in the NCBI BioProject repository PRJNA717139. Further, Candida genomes are also available via https://ggkbase.berkeley.edu/project_groups/candida_genomes (sign in as a user is required).

\section{Declarations}

Ethics approval and consent to participate

This study was reviewed and approved by the University of Pittsburgh Institutional Review Board (IRB PR012100487 and PRO10090089).

Consent for publication

Not applicable

\section{Competing interests}

The authors declare that they have no competing interests.

\section{Author details}

${ }^{1}$ Department of Plant and Microbial Biology, University of California, Berkeley, CA, USA. ${ }^{2}$ Graduate School of Genome Science and Technology, The University of Tennessee, Knoxville, TN, USA. ${ }^{3}$ Chemical Sciences Division, Oak Ridge National Laboratory, Oak Ridge, TN, USA. ${ }^{4}$ Department of Microbiology and Immunology, Stanford University School of Medicine, Stanford, CA 94305, USA. ${ }^{5}$ Chan Zuckerberg Biohub, San Francisco, CA, USA. ${ }^{6}$ Department of Microbiology and Immunology, University of California, San Francisco, San Francisco, CA, USA. ${ }^{7}$ Department of Surgery, University of Pittsburgh School of Medicine, Pittsburgh, PA, USA. ${ }^{8}$ Division of Newborn Medicine, Magee-Womens Hospital of UPMC, Pittsburgh, PA, USA. 'Department of Earth and Planetary Science, University of California, Berkeley, CA, USA.

${ }^{10}$ Department of Environmental Science, Policy, and Management, University of California, Berkeley, CA, USA. ${ }^{11}$ Earth Sciences Division, Lawrence Berkeley National Laboratory, Berkeley, CA, USA

Received: 2 February 2021 Accepted: 22 April 2021

Published online: 21 June 2021

\section{References}

1. Naglik JR, Fidel PL Jr, Odds FC. Animal models of mucosal Candida infection. FEMS Microbiol Lett. 2008;283(2):129-39. https://doi.org/10.1111/ j.1574-6968.2008.01160.x

2. Silva S, Negri M, Henriques M, Oliveria R, Williams DW, Azeredo J. Candida glabrata. Candida parapsilosis, and Candida tropicalis: biology, epidemiology, pathogenicity, and antifungal resistance. FEMS Microbiol Rev. 2012:36(2):288-305. https://doi.org/10.1111/j.1574-6976.2011.00278.x.

3. Clerihew L, Lamagni TL, Brocklehurst P, McGuire W. Candida parapsilosis infection in very low birthweight infants. Arch Dis Child Fetal Neonatal Ed. 2007;92(2):127-9. https://doi.org/10.1136/fnn.2006.097758.

4. Bliss JM. Candida parapsilosis: an emerging pathogen developing its own identity. Virulence. 2015;6(2):109-11. https://doi.org/10.1080/21505594.2015.1 008897

5. Kuhn DM, Mikherjee PK, Clark TA, Pujol C, Chandra J, Hajjeh RA, et al. Candida parapsilosis characterization in an outbreak setting. Emerg Infect Dis. 2004;10(6):1074-81. https://doi.org/10.3201/eid1006.030873.

6. Trofa D, Gácser A, Nosanchuk JD. Candida parapsilosis, an emerging fungal pathogen. Clin Microbiol Rev. 2008;21(4):606-25. https://doi.org/10.1128/ CMR.00013-08.

7. Gonia S, Archambault L, Shevik M, Altendahl M, Fellows E, Bliss JM, et al. Candida parapsilosis protects premature intestinal epithelial cells from invasion and damage by Candida albicans. Front Pediatr. 2017;5:54. https:// doi.org/10.3389/fped.2017.00054.

8. Huang YC, Li CC, Lin TY, Chou YH, Wu JL, Hsueh C. Association of fungal colonization and invasive disease in very low birth weight infants. Pediatr 
Infect Dis J. 1998;17(9):819-22. https://doi.org/10.1097/00006454-19980900000014.

9. Fridkin SK, Kaufman D, Edwards JR, Shetty S, Horan T. Changing incidence of Candida bloodstream infections among NICU patients in the United States: 1995-2004. Pediatrics. 2006;117(5):1680-7. https://doi.org/10.1542/ peds.2005-1996.

10. Whaley SG, Berkow EL, Rybak JM, Nishimoto AT, Barker KS, Rogers PD. Azole antifungal resistance in Candida albicans and emerging non-albicans Candida species. Front Microbiol. 2017;7:2173. https://doi.org/10.3389/ fmicb.2016.02173.

11. Forsberg K, Woodworth K, Walters M, Berkow EL, Jackson B, Chiller T, et al. Candida auris: the recent emergence of a multidrug-resistant fungal pathogen. Med Mycol. 2019;57(1):1-12. https://doi.org/10.1093/mmy/ myy054.

12. Hibberd DJ. Observations on the ultrastructure of the choanoflagellate Codosiga botrytis (Ehr.) Saville-Kent with special reference to the flagellar apparatus. J Cell Sci. 1975;17(1):191-219. https://doi.org/10.1242/jcs.17.1.191.

13. Cantley AM, Woznica A, Beemelmanns C, King N, Clardy J. Isolation and synthesis of a bacterially produced inhibitor of rosette development in choanoflagellates. J Am Chem Soc. 2016;138(13):4326-9. https://doi.org/10.1 021/jacs.6b01190.

14. Woznica A, Gerdt JP, Hulett RE, Clardy J, King N. Mating in the closest living relatives of animals is induced by a bacterial chondroitinase. Cell. 2017; 170(6):1175-83. https://doi.org/10.1016/j.cell.2017.08.005.

15. Cruz MR, Graham CE, Gagliano BC, Lorenz MC, Garsin DA. Enterococcus faecalis inhibits hyphal morphogenesis and virulence of Candida albicans. Infect Immun. 2013;81(1):189-200. https://doi.org/10.1128/IAl.00914-12.

16. Bachtiar EW, Dewiyani S, Akbar SMS, Bachtiar BM. Inhibition of Candida albicans biofilm development by unencapsulated Enterococcus faecalis cps2. J Dental Sci. 2016;11(3):323-30. https://doi.org/10.1016/j.jds.2016.03.012.

17. West PT, Probst AJ, Grigoriev IV, Thomas BC, Banfield JF. Genomereconstruction for eukaryotes from complex natural microbial communities. Genome Res. 2018;28(4):569-80. https://doi.org/10.1101/gr.228429.117.

18. Olm MR, West PT, Brooks B, Firek BA, Baker R, Morowitz MJ, et al. Genomeresolved metagenomics of eukaryotic populations during early colonization of premature infants and in hospital rooms. Microbiome. 2019;7(1):26. https://doi.org/10.1186/s40168-019-0638-1.

19. Whaley SG, Tsao S, Weber S, Zhang Q, Barker KS, Raymond M, et al. The RTA3 Gene, Encoding a putative lipid translocase, influences the susceptibility of Candida albicans to fluconazole. Antimicrob Agents Chemother. 2016;60(10):6060-6. https://doi.org/10.1128/AAC.00732-16.

20. Afshinnekoo E, Meydan C, Chowdhury S, Jaroudi D, Boyer C, Bernstein N, et al. Geospatial resolution of human and bacterial diversity with city-scale metagenomics. Cell Syst. 2015;1 (1):97-97.e3. https://doi.org/10.1016/j.cels.2 015.07.006.

21. Olm MR, Bhattacharya N, Crits-Christoph A, Firek BA, Baker R, Song YS, et al. Necrotizing enterocolitis is preceded by increased gut bacterial replication, Klebsiella, and fimbriae-encoding bacteria. Sci Adv. 2019;5(12):eaax5727. https://doi.org/10.1126/sciadv.aax5727.

22. Butler $G$, Rasmussen M, Lin M, et al. Evolution of pathogenicity and sexual reproduction in eight Candida genomes. Nature. 2009;459:657-62. https:// doi.org/10.1038/nature08064.

23. Pryszcz LP, Németh T, Gácser A, Gabaldón T. Unexpected genomic variability in clinical and environmental strains of the pathogenic yeast Candida parapsilosis. Genome Biol Evol. 2013;5(12):2382-92 https://doi.org/1 0.1093/gbe/evt185

24. Magwene PM, Kayıkçı Ö, Granek JA, Reininga JM, Scholl Z, Murray D. Outcrossing, mitotic recombination, and life-history trade-offs shape genome evolution in Saccharomyces cerevisiae. PNAS. 2011;108(5):1987-92 https://doi.org/10.1073/pnas.1012544108.

25. Roller RK, Stoddard SF, Schmidt TM. Exploiting rRNA Operon Copy Number to Investigate Bacterial Reproductive Strategies. Nat Microbiol. 2016;1(11): 16160. https://doi.org/10.1038/nmicrobiol.2016.160.

26. Guida A, Lindstädt C, Maguire SL, Ding C, Higgins DG, Corton NJ, et al. Using RNA-seq to determine the transcriptional landscape and the hypoxic response of the pathogenic yeast Candida parapsilosis. BMC Genomics. 2011;12(1):628 https://doi.org/10.1186/1471-2164-12-628.

27. Nobile CJ, Johnson AD. Candida albicans Biofilms and Human Disease. Annu Rev Microbiol. 2015;69(1):71-92 https://doi.org/10.1146/annurev-micro-091 014-104330.Candida.
28. Nobile CJ, Fox EP, Nett JE, Sorrells TR, Mitrovich QM, Hernday AD, et al. A recently evolved transcriptional network controls biofilm development in Candida albicans. Cell. 2012;148(1-2):126-38 https://doi.org/10.1016/j.cell.2 011.10.048.

29. Sher Y, Olm MR, Raveh-Sadka T, Brown CT, Sher R, Firek B, et al. Combined analysis of microbial metagenomic and metatranscriptomic sequencing data to assess in situ physiological conditions in the premature infant gut. PLoS One. 2020;15(3):e0229537 https://doi.org/10.1371/journal.pone.0229537.

30. Beggs JD. Lsm proteins and RNA processing. Biochem Soc Trans. 2005;33(3): 433-8. https://doi.org/10.1042/BST0330433.

31. Desai JV, Lionakis MS. The role of neutrophils in host defense against invasive fungal infections. Curr Clin Microbiol Rep. 2018;5(3):181-9. https:// doi.org/10.1007/s40588-018-0098-6.

32. Mesquite-Rocha S, Godoy-Martinez PC, Gonçalves SS, Urrutia MD, Carlesse F, Seber A, et al. The water supply system as a potential source of fungal infection in paediatric haematopoietic stem cell units. BMC Infect Dis. 2013; 13(1):289. https://doi.org/10.1186/1471-2334-13-289.

33. Chang HJ, Miller HL, Watkins N, Arduino MJ, Ashford DA, Midgley G, et al. An epidemic of Malassezia pachydermatis in an intensive care nursery associated with colonization of helath care workers' pet dogs. N Engl J Med. 1998:338(11):706-11. https://doi.org/10.1056/NEJM199803123381102.

34. Pajic P, Pavlidis P, Dean K, Neznanova L, Romano R, Garneau D, et al. Independent amylase gene copy number bursts correlate with dietary preferences in mammals. eLife. 2019;8:e44628 https://doi.org/10.7554/ elife.44628.

35. Tuch BB, Mitrovich QM, Homann OR, Hernday AD, Monighetti CK, de La Vega FM, et al. The transcriptomes of two heritable cell types illuminate the circuit governing their differentiation. PLoS Genet. 2010;6(8):e1001070 https://doi.org/10.1371/journal.pgen.1001070.

36. Slutsky B, Staebell M, Anderson J, Risen L, Pfaller M, Soll DR. "White-opaque transition": a second high -frequency switching system in Candida albicans. J Bacteriol. 1987;169(1):189-97 https://doi.org/10.1128/jb.169.1.189-197.1987.

37. Miller MG, Johnson AD. White-opaque switching in Candida albicans is controlled by mating-type locus homeodomain proteins and allows efficient mating. Cell. 2002;110(3):293-302 https://doi.org/10.1016/s00928674(02)00837-1.

38. Ramirez-Zavala B, Reuss O, Park YN, Ohlsen K, Morschhäuser J. Environmental induction of white-opaque switching in Candida albicans. PLoS Pathog. 2008; 4(6):e1000089 https://doi.org/10.1371/journal.ppat.1000089.

39. Huang G, Srikantha T, Sahni N, Yi S, Soll DR. $\mathrm{CO}_{2}$ regulates white-to-opaque switching in Candida albicans. Curr Biol. 2009;19(4):330-4. https://doi.org/1 0.1016/j.cub.2009.01.018.

40. Haider S, Pal R. Integrated analysis of transcriptomic and proteomic data; 2013. p. 91-110.

41. Gong Y, Li T, Yu C, Sun S. Candida albicans heat shock proteins and hspsassociated signaling pathways as potential antifungal targets. Front Cell Infect Microbiol. 2017;7:520. https://doi.org/10.3389/fcimb.2017.00520.

42. Meng Q, Zhang T, Wei W, Mu W, Miao M. Production of Mannitol from a High Concentration of Glucose by Candida parapsilosis SK26.001. Appl Biochem Biotechnol. 2017;181(1):391-406. https://doi.org/10.1007/s12010-016-2219-0.

43. Dominguez EG, Zarnowski R, Choy HL, Zhao M, Sanchez H, Nett JE, et al. Conserved role for biofilm matrix polysaccharides in Candida auris drug resistance. mSphere. 2019:4(1):e00680-18. https://doi.org/10.1128/ mSphereDirect.00680-18.

44. Quiloan MLG, Vu J, Carvalho J. Enterococcus faecalis can be distinguished from Enterococcus faecium via differential susceptibility to antibiotics and growth and fermentation characteristics on mannitol salt agar. Front Biol. 2012;7(2):167-77. https://doi.org/10.1007/s11515-012-1183-5.

45. Guimaraes JC, Zavolan M. Patterns of ribosomal protein expression specify normal and malignant human cells. Genome Biol. 2016;17(1):236. https://doi. org/10.1186/s13059-016-1104-Z.

46. Shi Z, Fujii K, Kovary KM, Genuth NR, Röst HL, Teruel MN, et al. Heterogeneous ribosomes preferentially translate distinct subpools of mRNAs genome-wide. Mol Cell. 2017;67(1):71-83. https://doi.org/10.1016/j. molcel.2017.05.021.

47. Genuth NR, Barna M. The discovery of ribosome heterogeneity and its implications for gene regulation and organismal life. Mol Cell. 2018;71(3): 364-74. https://doi.org/10.1016/.molcel.2018.07.018Get.

48. De la Cruz J, Gómez-Herreros F, Rodríguez-Galán O, Begley V, de la Cruz Muñoz-Centeno M, Chávez S. Feedback regulation of ribosome assembly. Curr Genet. 2018;64(2):393-404. https://doi.org/10.1007/s00294-017-0764-x. 
49. Zhou X, Liao WJ, Liao JM, Liao P, Lu H. Ribosomal proteins: functions beyond the ribosome. J Mol Cell Biol. 2015;7(2):92-104. https://doi.org/10.1 093/jmcb/mjv014.

50. Cavalheiro M, Teixeira MC. Candida Biofilms: Threats, Challenges, and Promising Strategies. Front Med (Lausanne). 2018;13(5):28. https://doi.org/1 0.3389/fmed.2018.00028.

51. Mavor AL, Thewes S, Hube B. Systemic fungal infections caused by Candida species: epidemiology, infection process and virulence attributes. Curr Drug Targets. 2005;6(8):863-74. https://doi.org/10.2174/138945005774912735.

52. Brown CT, Xiong W, Olm MR, Thomas BC, Baker R, Firek B, et al. Hospitalized premature infants are colonized by related bacterial strains with distinct proteomic profiles. MBio. 2018;9:e00441-18.

53. Brooks B, Olm MR, Firek BA, Baker R, Thomas BC, Morowitz MJ, et al. Strainresolved analysis of hospital rooms and infants reveals overlap between the human and room microbiome. Nat Commun. 2017;8(1):1814. https://doi. org/10.1038/s41467-017-02018-w

54. Raveh-Sadka T, Thomas BC, Singh A, Firek B, Brooks B, Castelle CJ, et al. Gut bacteria are rarely shared by co-hospitalized premature infants, regardless of necrotizing enterocolitis development. Elife. 2015;4:e05477. https://doi.org/1 0.7554/eLife.05477.

55. Rahman SF, Olm MR, Morowitz MJ, Banfield JF. Machine learning leveraging genomes from metagenomes identifies influential antibiotic resistance genes in the infant gut microbiome. mSystems. 2018;3:e00123-17.

56. Sharon I, Morowitz MJ, Thomas BC, Costello EK, Relman DA, Banfield JF. Time series community genomics analysis reveals rapid shifts in bacterial species, strains, and phage during infant gut colonization. Genome Res. 2013;23(1):111-20. https://doi.org/10.1101/gr.142315.112.

57. Brooks B, Olm MR, Firek BA, Baker R, Geller-McGrath D, Reimer SR, et al. The developing premature infant gut microbiome is a major factor shaping the microbiome of neonatal intensive care unit rooms. Microbiome. 2018;6:112.

58. Peng Y, Leung HCM, Yiu SM, Chin FYL. IDBA-UD: a de novo assembler for single-cell and metagenomic sequencing data with highly uneven depth. Bioinformatics. 2012;28(11):1420-8. https://doi.org/10.1093/bioinformatics/ bts174.

59. Alneberg J, Bjarnason BS, Brujin I, Schirmer M, Quick J, ljaz UZ, et al. Binning metagenomic contigs by coverage and composition. Nat Methods. 2014; 11(11):1144-6. https://doi.org/10.1038/nmeth.3103.

60. Ter-Hovhannisyan V, Lomsadze A, Chernoff YO, Borodovsky M. Gene prediction in novel fungal genomes using an ab initio algorithm with unsupervised training. Genome Res. 2008;18(12):1979-90. https://doi.org/1 $0.1101 /$ gr.081612.108.

61. Stanke M, Keller O, Gunduz I, Hayes A, Waack S, Morgenstern B. AUGUSTUS: ab initio prediction of alternative transcripts. Nucleic Acids Res. 2006;34(Web Server):W435-9. https://doi.org/10.1093/nar/gkl200.

62. Simão FA, Waterhouse RM, loannidis P, Kriventseva EV, Zdobnov EM BUSCO: assessing genome assembly and annotation completeness with single-copy orthologs. Bioinformatics. 2015;31(19):3210-2. https://doi.org/1 0.1093/bioinformatics/btv351

63. Cantarel BL, Korf I, Robb SMC, Parra G, Ross E, Moore B, et al. MAKER: an easy-to-use annotation pipeline designed for emerging model organism genomes. Genome Res. 2008;18(1):188-96. https://doi.org/10.1101/gr.6743 907.

64. Langmead B, Salzberg SL. Fast gapped-read alignment with Bowtie 2. Nat Methods. 2012;9(4):357-9. https://doi.org/10.1038/nmeth.1923.

65. Quinlan AR, Hall IM. BEDTools: a flexible suite of utilities for comparing genomic features. Bioinformatics. 2010;26(6):841-2. https://doi.org/10.1093/ bioinformatics/btq033.

66. Lee TH, Guo H, Wang X, Kim C, Paterson AH. SNPhylo: a pipeline to construct a phylogenetic tree from huge SNP data. BMC Genomics. 2014; 15(1):162. https://doi.org/10.1186/1471-2164-15-162.

67. Olm MR, Brown CT, Brooks B, Banfield JF. dRep: a tool for fast and accurate genomic comparisons that enables improved genome recovery from metagenomes through de-replication. ISME J. 2017;11(12):2864-8. https:// doi.org/10.1038/ismej.2017.126.

68. Purcell S, Neale B, Todd-Brown K, Thomas L, Ferreira MAR, Bender D, et al. PLINK: a toolset for whole-genome association and population-based linkage analysis. Am J Hum Genet. 2007;81:559-75.

69. Alexander DH, Lange K. Enhancements to the ADMIXTURE algorithm for individual ancestry estimation. BMC Bioinformatics. 2011;12(1):246. https:// doi.org/10.1186/1471-2105-12-246.
70. Robinson JT, Thorvaldsdóttir H, Wenger AM, Zehir A, Mesirov JP. Variant Review with the Integrative Genomics Viewer (IGV). Cancer Res. 2017;77(21): $31-4$.

71. Bray NL, Pimental H, Melsted P, Pachtor L. Near-optimal probabilistic RNAseq quantification. Nat Biotechnol. 2016;34(5):525-7. https://doi.org/10.1038/ nbt.3519.

72. Love Ml, Huber W, Anders S. Moderated estimation of fold change and dispersion for RNA-seq data with DESeq2. Genome Biol. 2014;15(12):550. https://doi.org/10.1186/s13059-014-0550-8.

73. Rohart F, Gautier B, Singh A, Cao KL. mixOmics: An R package for 'omics feature selection and multiple data integration. PLoS Comput Biol. 2017; 13(11):e1005752. https://doi.org/10.1371/journal.pcbi.1005752.

74. Aramaki T, Blanc-Mathieu R, Endo H, Ohkubo K, Kanehisa M, Goto S, et al. KofamKOALA: KEGG ortholog assignment based on profile HMM and adaptive score threshold. Bioinformatics. 2020;36(7):2251-2.

75. El-Gebali S, Mistry J, Bateman A, Eddy SR, Luciani A, Potter SC, et al. The PFAM protein families database in 2019. Nucleic Acids Res. 2019;47(D1): D427-32. https://doi.org/10.1093/nar/gky995.

76. Core Team R. R: A language and environment for statistical computing. Vienna: R Foundation for Statistical Computing; 2013. http://www.R-project. org/

77. Dorfer V, Maltsev S, Winkler S, Mechtler K. CharmeRT: Boosting peptide identifications by chimeric spectra identification and retention time prediction. J Proteome Res. 2018;17(8):2581-9. https://doi.org/10.1021/acs. jproteome.7b00836.

78. Edgar RC. Search and clustering orders of magnitude faster than BLAST Bioinformatics. 2010;26(19):2460-1. https://doi.org/10.1093/bioinformatics/ btq461.

79. Polpitiya AD, Qian WJ, Jaitly N, Petyuk VA, Adkins JN, Camp DG, et al. DAnTE: a statistical tool for quantitative analysis of-omics data. Bioinformatics. 2008;24(13):1556-8. https://doi.org/10.1093/bioinformatics/ btn217.

80. Tyanova S, Temu T, Sinitcyn P, Carlson A, Hein MY, Geiger T, et al. The Perseus computational platform for comprehensive analysis of (prote) omics data. Nat Methods. 2016;13(9):731-40. https://doi.org/10.1038/nmeth.3901.

81. Croft D, O'Kelly G, Wu G, Haw R, Gillespie M, et al. Reactome: a database of reactions, pathways and biological processes. Nucleic Acids Res. 2011. 39(Database issue):D691-7. https://doi.org/10.1093/nar/gkq1018. Epub 2010 Nov 9.

\section{Publisher's Note}

Springer Nature remains neutral with regard to jurisdictional claims in published maps and institutional affiliations.

Ready to submit your research? Choose BMC and benefit from:

- fast, convenient online submission

- thorough peer review by experienced researchers in your field

- rapid publication on acceptance

- support for research data, including large and complex data types

- gold Open Access which fosters wider collaboration and increased citations

- maximum visibility for your research: over $100 \mathrm{M}$ website views per year

At $\mathrm{BMC}$, research is always in progress.

Learn more biomedcentral.com/submission 


\section{University Library}

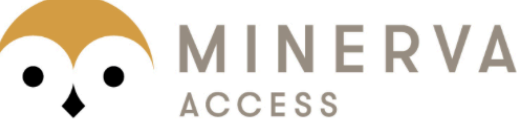

A gateway to Melbourne's research publications

Minerva Access is the Institutional Repository of The University of Melbourne

Author/s:

West, PT;Peters, SL;Olm, MR;Yu, FB;Gause, H;Lou, YC;Firek, BA;Baker, R;Johnson, AD;Morowitz, MJ;Hettich, RL;Banfield, JF

Title:

Genetic and behavioral adaptation of Candida parapsilosis to the microbiome of hospitalized infants revealed by in situ genomics, transcriptomics, and proteomics

Date:

2021-06-21

Citation:

West, P. T., Peters, S. L., Olm, M. R., Yu, F. B., Gause, H., Lou, Y. C., Firek, B. A., Baker, R., Johnson, A. D., Morowitz, M. J., Hettich, R. L. \& Banfield, J. F. (2021). Genetic and behavioral adaptation of Candida parapsilosis to the microbiome of hospitalized infants revealed by in situ genomics, transcriptomics, and proteomics. MICROBIOME, 9 (1), https:// doi.org/10.1186/s40168-021-01085-y.

Persistent Link:

http://hdl.handle.net/11343/296183

License:

CC BY 